Clinical Research

\title{
Are adipokines the missing link between obesity, immune response, and outcomes in severe COVID-19?
}

\author{
Mathieu Blot $\mathbb{1}^{1,2,3,4} \cdot$ David Masson 2,3,4,5$\cdot$ Maxime Nguyen ${ }^{2,3,4,6} \cdot$ Abderrahmane Bourredjem ${ }^{7,8} \cdot$ LYMPHONIE Study \\ Group $\cdot$ Christine Binquet ${ }^{2,3,4,7,8} \cdot$ Lionel Piroth $^{1,7,8}$
}

Received: 4 December 2020 / Revised: 1 May 2021 / Accepted: 18 May 2021 / Published online: 31 May 2021

(c) The Author(s), under exclusive licence to Springer Nature Limited 2021

\begin{abstract}
Introduction Obesity is commonly reported in COVID-19 patients and is associated with poorer outcomes. It is suggested that leptin could be the missing link between obesity and severe COVID-19. Our study aimed to unravel the link between adipokines, COVID-19 status, immune response, and outcomes in severe pneumonia.

Methods In this prospective observational single-center study, 63 immunocompetent patients with severe pneumonia (36 non-COVID-19 and 27 COVID-19) were enrolled, most required intensive care. Clinical and biological characteristics (glucose metabolism, plasma adipokines, and cytokine concentrations) and outcomes were compared.

Results At similar baseline severity, COVID-19 patients required mechanical ventilation for significantly longer than nonCOVID-19 patients $(p=0.0049)$. Plasma concentrations of leptin and adiponectin were respectively positively and negatively correlated with BMI and glucose metabolism (glycemia and insulinemia), but not significantly different between the two groups. Leptin levels were negatively correlated with IL-1 $\beta$ and IL-6, but the adipokines were not correlated with most other inflammatory mediators, baseline severity (SOFA score), or the duration of mechanical ventilation.

Conclusion Adipokine levels were correlated with BMI but not with most inflammatory mediators, severity, or outcomes in severe pneumonia, regardless of the origin. The link between obesity, dysregulated immune response, and life-threatening COVID-19 requires further investigation.

Clinical trial ClinicalTrials.gov: NCT03505281.A list of authors and their affiliations appears at the end of the paper.
\end{abstract}

Mathieu Blot

mathieu.blot@chu-dijon.fr

1 Infectious Diseases Department, Dijon Bourgogne University Hospital, Dijon, France

2 Université Bourgogne Franche-Comté, Dijon, France

3 INSERM, LNC UMR 1231, Dijon, France

4 FCS Bourgogne-Franche Comté, LipSTIC LabEx, Dijon, France

5 Laboratory of Clinical Chemistry, Dijon Bourgogne University Hospital, Dijon, France

6 Anesthesiology and Critical Care Department, Dijon Bourgogne University Hospital, Dijon, France

7 Clinical Epidemiology Unit, INSERM, CIC1432, Dijon, France

8 Clinical Epidemiology/Clinical Trials Unit, Clinical Investigation Center, Dijon Bourgogne University Hospital, Dijon, France

\section{Introduction}

COVID-19 has peculiar characteristics and outcomes, suggesting a unique immunopathogenesis. Overweight and obesity are associated with significant increases in morbidity and mortality from COVID-19, namely an increased susceptibility to COVID-19, and related hospitalization, ICU admission, and mortality [1-4]. Increased susceptibility to COVID-19 severity has been linked to associated comorbidities as cardiovascular diseases and diabetes. However, obesity, in its own right, is considered a metabolic disease including insulin resistance, glucose metabolism, and adipokines alterations, which could also explain such features $[1,3]$. It has been suggested for example that leptin overproduction in individuals with obesity could be the missing link between obesity and dysregulated immune response and outcomes in severe COVID-19 [5]. Leptin, which is secreted proportionally to adipocyte mass, is a critical regulator of the immune response, increasing Th-1 response, monocytes/ macrophages activation, and pro-inflammatory cytokines 
Table 1 Baseline characteristics and outcomes of the study population (LYMPHONIE study, 2018-2020).

\begin{tabular}{|c|c|c|c|}
\hline & \multicolumn{2}{|l|}{ Study group } & \multirow[t]{2}{*}{$p$} \\
\hline & $\begin{array}{l}\text { Non-COVID-19 } \\
N=36\end{array}$ & $\begin{array}{l}\text { COVID-19 } \\
N=27\end{array}$ & \\
\hline \multicolumn{4}{|l|}{ Demographics } \\
\hline Age (years), median (IQR) & $67.5(63-76.5)$ & $64(57-71)$ & 0.06 \\
\hline Male sex, $n(\%)$ & $29(81 \%)$ & $17(63 \%)$ & 0.12 \\
\hline Body mass index $\left(\mathrm{kg} / \mathrm{m}^{2}\right)$, median (IQR) & $27(25-33)$ & $31(27-34)$ & 0.30 \\
\hline \multicolumn{4}{|l|}{ Chronic comorbidities } \\
\hline Cardiovascular disease, $n(\%)$ & $12(33 \%)$ & $5(19 \%)$ & 0.25 \\
\hline Pulmonary disease, $n(\%)$ & $12(33 \%)$ & $5(19 \%) 28$ & 0.25 \\
\hline Chronic renal disease, $n(\%)$ & $2(6 \%)$ & $1(4 \%)$ & 0.73 \\
\hline Cerebrovascular disease, $n(\%)$ & $5(14 \%)$ & $3(11 \%)$ & 0.74 \\
\hline Diabetes mellitus, $n(\%)$ & $10(28 \%)$ & $2(7 \%)$ & 0.28 \\
\hline Charlson score, mean $\pm \mathrm{SD}$ & $1.5 \pm 2.0$ & $0.9 \pm 0.9$ & 0.12 \\
\hline \multicolumn{4}{|l|}{ Severity at hospital admission } \\
\hline Septic shock, $n(\%)$ & $11(31 \%)$ & 0 & 0.0015 \\
\hline ARDS, $n(\%)$ & $23(64 \%)$ & $25(93 \%)$ & 0.015 \\
\hline Pneumonia Severity Index, mean \pm SD & $117.8 \pm 38.6$ & $94.2 \pm 27.1$ & 0.006 \\
\hline SOFA score, mean \pm SD & $7.2 \pm 3.6$ & $6.7 \pm 2.0$ & 0.52 \\
\hline \multicolumn{4}{|l|}{ Biological findings at admission } \\
\hline ASAT (IU/l), mean \pm SD & $86.3 \pm 92.4$ & $86.2 \pm 54.6$ & 0.99 \\
\hline Serum creatinine $(\mu \mathrm{mol} / \mathrm{l})$, mean $\pm \mathrm{SD}$ & $132.9 \pm 93.3$ & $90.2 \pm 40.7$ & 0.02 \\
\hline $\mathrm{PaO}_{2}: \mathrm{FiO}_{2}(\mathrm{~mm} \mathrm{Hg})$, mean $\pm \mathrm{SD}$ & $123.7 \pm 54.9$ & $136.2 \pm 49.8$ & 0.35 \\
\hline Lactate level $(\mathrm{mmol} / \mathrm{l})$, mean \pm SD & $2.6 \pm 1.9$ & $1.7 \pm 0.7$ & 0.01 \\
\hline \multicolumn{4}{|l|}{ Glucose metabolism and adipokines } \\
\hline Glycemia $(\mathrm{mmol} / \mathrm{l})$, mean \pm SD & $16 \pm 8$ & $12 \pm 6$ & 0.024 \\
\hline Insulin $(\mathrm{mU} / \mathrm{l})$, mean $\pm \mathrm{SD}$ & $15 \pm 25$ & $13 \pm 13$ & 0.63 \\
\hline Leptin (ng/ml), mean \pm SD & $18.5 \pm 27.5$ & $15.0 \pm 19.1$ & 0.58 \\
\hline Adiponectin (ng/ml), mean \pm SD & $5338 \pm 4024$ & $4090 \pm 2745$ & 0.18 \\
\hline Adiponectin/Leptin ratio & $1.5 \pm 2.1$ & $1.2 \pm 2.9$ & 0.61 \\
\hline \multicolumn{4}{|l|}{ Outcomes at 30 days } \\
\hline Median days of mechanical ventilation (IQR) & $4(0-15)$ & $15(7-22)$ & 0.0049 \\
\hline Median hospital length of stay (days) (IQR) & $21(13-30)$ & $29(20-30)$ & 0.087 \\
\hline 30-day mortality, $n(\%)$ & $2(6 \%)$ & $1(4 \%)$ & 1 \\
\hline
\end{tabular}

release, three hallmarks of the immune response associated with critical COVID-19 [1, 6, 7]. Our study aimed to unravel the link between plasma adipokines (leptin and adiponectin), COVID-19 status, immune response, and outcomes in severe pneumonia, either related to SARS-CoV-2 or not.

\section{Methods}

We performed a prospective, exploratory, ancillary study of the ongoing LYMPHONIE project (NCT03505281) initiated in November 2018 at the University Hospital of DijonBourgogne (France) [8]. We included non-immunecompromised patients with severe pneumonia (at least two criteria of the quick-SOFA score and/or need for mechanical ventilation (MV) or vasopressors). COVID-19 patients all tested positive for SARS-CoV-2 by reverse transcriptase-polymerase chain reaction, and non-COVID19 were enrolled before the pandemic started in Burgundy (France). Approval was obtained from the ethics committee (Comité de Protection des Personnes SUD MEDITERRANEE V; 2017-A03404-49). Ethylenediaminetetraacetic acid anticoagulated blood was obtained after the inclusion of the patient and within $48 \mathrm{~h}$ of hospital admission. Clinical and biological parameters were recorded, and the SOFA score was calculated at the time of inclusion. The following clinical outcomes were recorded up to 30 days after admission: 30-day mortality, duration of MV. Leptin, adiponectin, and inflammatory cytokines and chemokines (C-C motif chemokine ligand (CCL) 2, C-X-C motif chemokine 
A

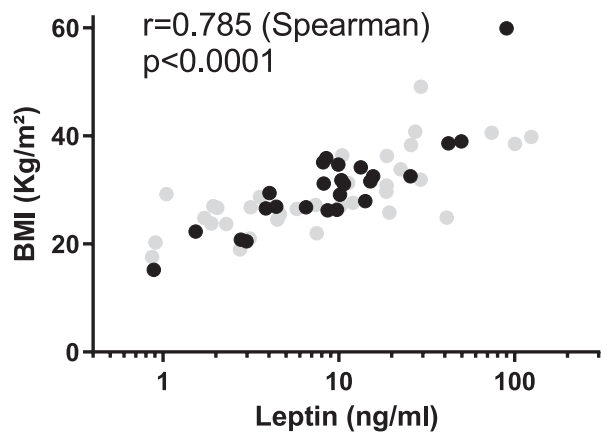

B

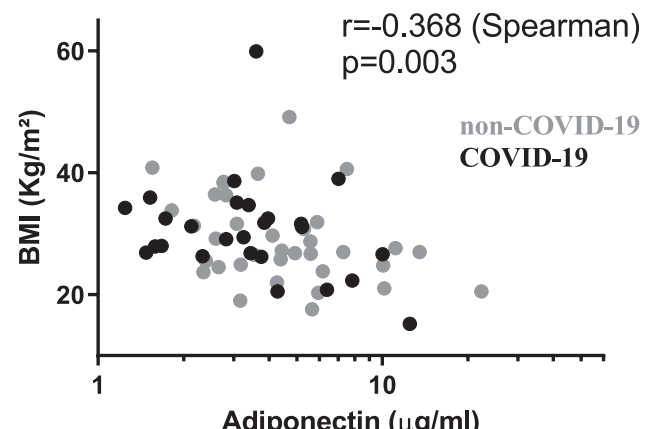

C

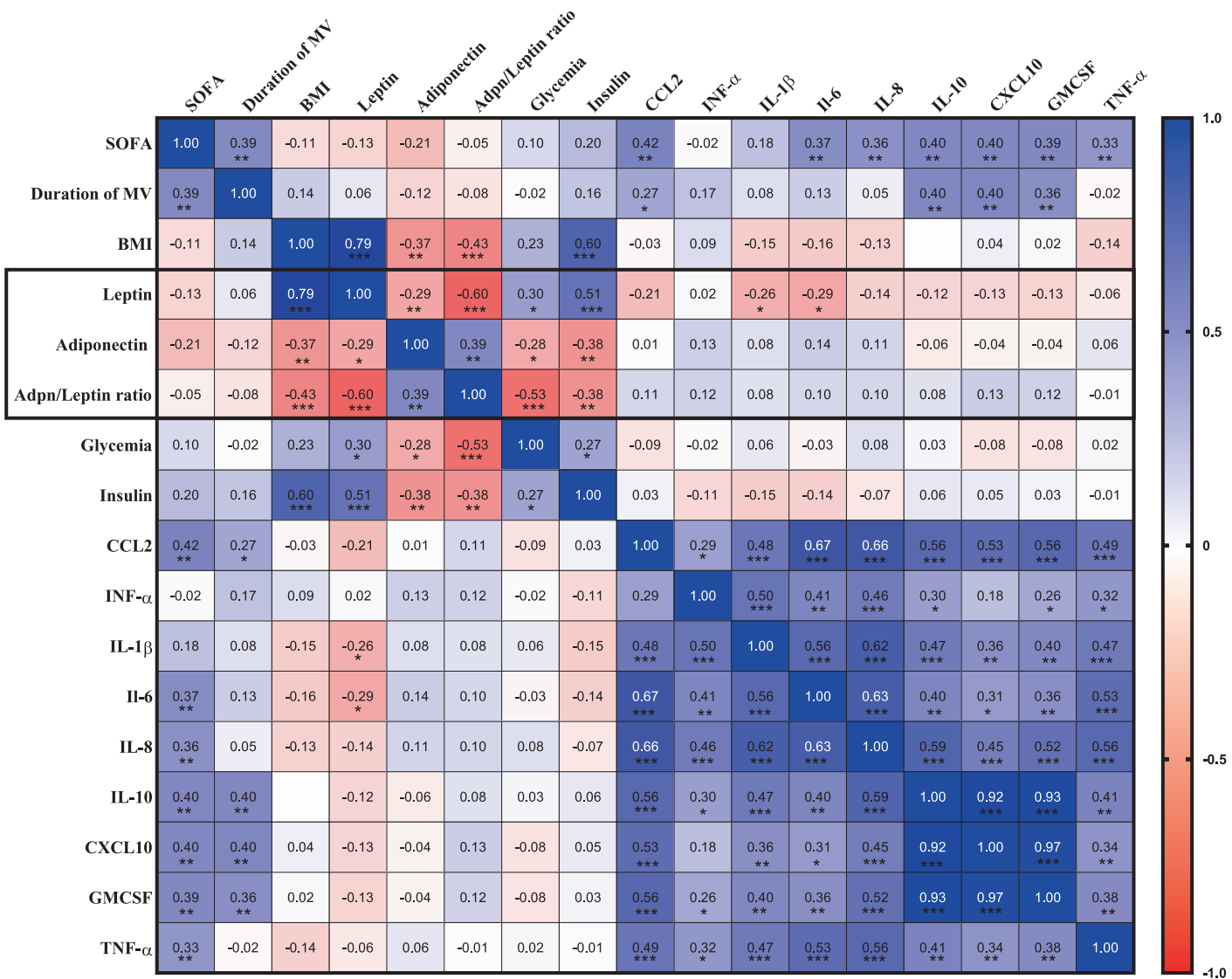

Fig. 1 Correlations between adipokines, body mass index, clinical severity and outcome, glucose metabolism, and plasma cytokine concentrations. Scatterplot depicting correlations between body mass index (BMI) and plasma concentrations of leptin (A) or adiponectin (B). (C) Heatmap of the Spearman correlation $(r)$ between adipokines,

ligand (CXCL) 10, granulocyte-macrophage colony-stimulating factor (GM-CSF), interferon (IFN)- $\alpha$, interleukin (IL)-1 $\beta$, IL-6, IL-8, IL-10, tumor necrosis factor (TNF)- $\alpha$ ) were quantified in plasma using the Magnetic Luminex assay (R\&D Systems, USA) according to the manufacturer's instructions [8]. Characteristics were described
BMI, clinical severity and outcome, glucose metabolism, and plasma cytokine concentrations. Spearman correlations: $* p<0.05$; $* * p<0.01$; $* * * p<0.001$ between each cytokine (our outcome) and ELF concentration of SARS-CoV-2 (LYMPHONIE study, 2018-2020).

according to COVID-19 status (i.e., non-COVID-19 vs COVID-19). Continuous variables were expressed as mean \pm standard deviation or medians and interquartile range (IQR) according to their distribution, and categorical variables as frequencies and percentages. Univariate comparisons were performed using Student's test for means, 
Wilcoxon Mann-Whitney test for medians and IQRs and $\chi^{2}$ test (or Fisher's exact test when appropriate) for percentages. Spearman correlations (as well as their 95\% confidence interval) were computed between adipokines, body mass index (BMI), glucose metabolism parameters, and the most pertinent clinical outcomes associated with COVID-19 status; correlation central estimates were depicted with a heatmap representation and scatter-plots. A $p$ value $<0.05$ was considered statistically significant. Analyses were performed using SAS version 9.4 (SAS Institute Inc., Cary, NC, USA).

\section{Results}

Thirty-six non-COVID-19 and 27 COVID-19 patients with severe pneumonia were enrolled. Median age was marginally lower in the COVID-19 group as compared to the nonCOVID-19 group (64 (57-71) vs. 67.5 (36-76.5); $p=$ 0.06). BMI was not significantly different between the two groups. Fewer COVID-19 patients had septic shock (0 vs. $31 \% ; p=0.0015)$, and arterial lactates $(1.7 \pm 0.7$ vs. $2.6 \pm$ $1.9 \mathrm{mmol} / 1 ; p=0.01)$, serum creatinine (90.2 \pm 40.7 vs. $132.9 \pm 93.3 \mu \mathrm{mol} / \mathrm{l} ; p=0.02$ ), and glycemia (12 \pm 6 vs. $16 \pm 8 \mathrm{mmol} / \mathrm{l} ; p=0.024)$ were all lower in COVID-19 patients. COVID-19 patients displayed similar plasma concentrations of leptin and adiponectin (respectively $15.0 \pm 19.1$ vs. $18.5 \pm 27.5 \mathrm{ng} / \mathrm{ml} ; p=0.58$ and $4090 \pm 2745$ vs. $5338 \pm 4024 \mathrm{ng} / \mathrm{ml} ; p=0.18)$, as compared to nonCOVID-19 patients. Although the $\mathrm{PaO}_{2}: \mathrm{FiO}_{2}$ ratio and SOFA score were not different between groups (136.2 \pm 49.8 vs. $123.7 \pm 54.9 \mathrm{~mm} \mathrm{Hg} ; p=0.35$ and $6.7 \pm 2.0$ vs. $7.2 \pm 3.6 ; \quad p=0.52$, respectively), COVID-19 patients required $\mathrm{MV}$ for significantly longer (median days 15 (7-22) vs. 4 (0-15); $p=0.0049)$ (Table 1).

Plasma concentrations of leptin were significantly positively correlated with the BMI $(r=0.785 ; p<0.0001)$, glycemia $(r=0.302 ; p=0.019)$, and insulinemia $(r=$ $0.511 ; p<0.001)$, while adiponectin levels were inversely correlated with BMI $(r=-0.368 ; p=0.003)$, glycemia $(r=-0.275 ; p=0.033)$, and insulinemia $(r=-0.376 ; p=$ 0.003 ) in patients with severe pneumonia. We observed an inverse correlation between leptin and IL-1 $\beta(r=-0.256$; $p=0.045)$ and IL-6 $(r=-0.287 ; p=0.024)$. No other significant correlations were observed between plasma adipokines and most inflammatory cytokines (CCL2, INF$\alpha$, IL-8, IL-10, CXCL10, GM-CSF, TNF- $\alpha$, all with $p>$ 0.10) (Fig. 1C). Plasma concentrations of leptin were not significantly correlated with baseline severity (SOFA score: $r=-0.127 ; 95 \% \mathrm{CI}=-0.371 ; 0.134 ; p=0.33)$ or the duration of MV $(r=0.063 ; 95 \% \mathrm{CI}=-0.197 ; 0.314$; $p=0.63$ ). Similarly, adiponectin concentrations were not significantly correlated with baseline severity (SOFA score: $r=-0.213 ; 95 \% \mathrm{CI}=-0.446 ; 0.046 ; p=0.10)$ or the duration of $\mathrm{MV}(r=-0.124 ; 95 \% \mathrm{CI}=-0.369 ; 0.137$; $p=0.34)$.

\section{Discussion}

Obesity was found to be a major factor for mortality during the H1N1 influenza pandemic; when compared to seasonal influenza, the effect of obesity appears to be even more significant in the ongoing COVID-19 pandemic [3-5, 9]. Several obesity-associated alterations such as metabolic changes, adipokine signaling, and epigenetic regulation may compromise immune response [5, 10]. Leptin concentrations were found to be higher in patients with COVID19 than in healthy people [11], and it has been suggested that leptin plays a critical role in the dysregulated immune response that leads to life-threatening COVID-19 [5]. However, such an association has not yet been studied to our knowledge. In obese patients, leptin levels are increased, and adiponectin levels are decreased, as we observed with the respective strong positive and negative correlations with BMI. Leptin is secreted by adipose tissue and acts as a central pro-inflammatory regulator of innate and adaptative immune responses, especially by increasing T-cell proliferation and Th-1 response [5]. Interestingly, we recently reported that both alveolar and plasma concentrations of CXCL10, a Th-1 chemokine, were higher in COVID-19 patients than in non-COVID-19 patients, and were independently associated with a longer duration of MV [8, 12]. Accordingly, we tried to unravel the link between adipokines and inflammatory cytokine production in patients with severe COVID-19. First, we found that plasma leptin and adiponectin levels were not significantly different between non-COVID-19 and COVID-19 patients. Next we observed an inverse correlation between leptin and IL-1 $\beta$ and IL-6, both of which were previously found to be significantly lower in COVID-19 patients than in non-COVID-19 patients $[8,12]$. The inverse correlation between plasma leptin and IL-6 was previously observed in an obese mouse model of acute lung injury and in a large cohort of ARDS patients as well, suggesting that hyperleptinemia could impair host immune response [13]. However, we observed no significant correlation between the two adipokines and most inflammatory mediators, especially those recently identified as the distinct cytokine signature observed in COVID-19 patients when compared to severe pneumonia of other origins (i.e., CXCL10, GM-CSF, and IL-10) [8, 14]. Finally, we observed no significant correlation between adipokines and baseline severity or the duration of $\mathrm{MV}$, which was twice as long in COVID-19 patients as in non-COVID-19 patients.

Here we confirmed that circulating levels of leptin and adiponectin are consistent biomarkers of obesity and 
glucose metabolism even in a context of severe pneumonia, as reflected by strong correlations we observed with BMI, glucose, and insulin concentrations, but with poor performance to predict the outcomes. The link between circulating adipokines, immune response, and outcomes deserve further research to unravel the potential role played to drive the dysregulated immune response observed in the most severe forms of COVID-19. We do not exclude a closer link with the pulmonary immune response, as previously reported [13], that we were unable to measure. However, several other hypotheses have been suggested to explain the link between obesity and life-threatening COVID-19. First, angiotensin-converting enzyme 2 receptor expression is upregulated in the bronchial epithelium of obese people [15]. In addition, type I and III IFN responses were found to be delayed and blunted in obese mice suffering from influenza infection, which could explain the ineffective viral clearance observed in obese patients [16]. Finally, it has been suggested that the overactivation of monocytes/macrophages contributes to a dysregulated immune response. Macrophages are present in adipose tissue and are thus increased in obesity [6]. They are likely to significantly contribute to metabolic dysfunction and immune overactivation in obese people with COVID-19 [17].

This study has several limitations. BMI was the only variable recorded to characterize the body composition of the patients included but not the most accurate metric to measure fat mass or visceral adipose tissue. Other metrics, such as waist circumference, skinfold thickness, would have been more accurate, but were not recorded prospectively in our study [1]. Given the small sample size, the heterogeneous distribution of comorbidities between the two groups, and the single-center design of our exploratory study, these results need to be confirmed in larger cohorts. However, this exploratory study was conducted in the context of a worldwide pandemic. The studies of adipokines in COVID-19 are still scarce in the literature, as are comparisons between non-COVID-19 and COVID-19 severe pneumonia, even though they are essential for understanding the distinctive pathogenesis of severe forms of COVID-19.

In conclusion, leptin and adiponectin levels were correlated with BMI but not with baseline severity, outcomes, and most inflammatory mediators in patients with severe pneumonia, regardless of whether it was COVID-19 or non-COVID-19. The link between adipokines, immune response, and lifethreatening COVID-19 is not as straightforward as expected. Further investigations are urgently required to understand the pathophysiological processes that make obese people more susceptible to severe forms of COVID-19.

\section{Data availability}

All data are available on demand.
Acknowledgements The authors thank all the investigators of the Lymphonie Study Group (Pascal Andreu, François Aptel, Jeremy Barben, Guillaume Beltramo, Philip Bielefeld, Philippe Bonniaud, Bernard Bonnotte, Belaid Bouhemad, Marielle Buisson, PierreEmmanuel Charles, Pascal Chavanet, Auguste Dargent, Hervé Devilliers, Thomas Gauthier, Marjolaine Georges, Alexandre Guilhem, Julien Guy, Marie Labruyère, Audrey Large, Serge Monier, Suzanne Mouries-Martin, Sébastien Prin, and Alain Putot). We thank the CIC-EC1432 (Alexandra Lamotte-Felin, Lydie Rossie, and Delphine Pecqueur), the Intensive Care Medicine Research team (Solenne Villot, Mathilde Audry, and Samantha Lima), the Cytometry Core Facility, Berengere Grammatico from the Laboratory of Virology and Shaliha Bechoua from the CRB (Centre de Ressource Biologique). We also thank all the donors (crowdfunding; https://thellie.org/covid-19), and the patients and healthcare team. We thank Suzanne Rankin for proofreading and editing the manuscript.

LYMPHONIE Study Group Pascal Andreu ${ }^{9}$, François Aptel ${ }^{9}$, Jeremy Barben $^{10}$, Guillaume Beltramo ${ }^{11}$, Philip Bielefeld ${ }^{12}$, Philippe Bonniaud $^{11}$, Bernard Bonnotte ${ }^{13}$, Belaid Bouhemad ${ }^{6}$, Marielle Buisson ${ }^{1}$, Pierre-Emmanuel Charles ${ }^{9}$, Pascal Chavanet ${ }^{1}$, Auguste Dargent ${ }^{9}$, Hervé Devilliers ${ }^{12}$, Thomas Gauthier ${ }^{2,3,4}$, Marjolaine Georges ${ }^{11}$, Alexandre Guilhem ${ }^{13}$, Julien Guy ${ }^{14}$, Marie Labruyère ${ }^{9}$, Audrey Large?, Serge Monier ${ }^{15}$, Suzanne Mouries-Martin ${ }^{12}$, Jean-Pierre Quenot ${ }^{9}$, Sébastien Prin ${ }^{9}$, Alain Putot ${ }^{10}$

${ }^{9}$ Department of Intensive Care, Dijon Bourgogne University Hospital, Dijon, France; ${ }^{10}$ Geriatrics Internal Medicine Department, Dijon Bourgogne University Hospital, Dijon, France; ${ }^{11}$ Department of Pneumology, Dijon Bourgogne University Hospital, Dijon, France; ${ }^{12}$ Department of Internal Medicine and Systemic Diseases, Dijon Bourgogne University Hospital, Dijon, France; ${ }^{13}$ Department of Internal Medicine and Clinical Immunology, Dijon Bourgogne University Hospital, Dijon, France; ${ }^{14}$ Hematobiology, Dijon Bourgogne University Hospital, Dijon, France; ${ }^{15}$ Cytometry Core Facility, University of Burgundy Franche-Comté, Dijon, France

${ }^{9}$ Department of Intensive Care, Dijon Bourgogne University Hospital, Dijon, France; ${ }^{10}$ Geriatrics Internal Medicine Department, Dijon Bourgogne University Hospital, Dijon, France; ${ }^{11}$ Department of Pneumology, Dijon Bourgogne University Hospital, Dijon, France; ${ }^{12}$ Department of Internal Medicine and Systemic Diseases, Dijon Bourgogne University Hospital, Dijon, France; ${ }^{13}$ Department of Internal Medicine and Clinical Immunology, Dijon Bourgogne University Hospital, Dijon, France; ${ }^{14}$ Hematobiology, Dijon Bourgogne University Hospital, Dijon, France; ${ }^{15}$ Cytometry Core Facility, University of Burgundy Franche-Comté, Dijon, France

Author contributions Concept and design: MB, DM, CB, LP; recruitment of patients: $\mathrm{MB}, \mathrm{MN}, \mathrm{LP}$; acquisition, analysis, or interpretation of data: $\mathrm{MB}, \mathrm{AB}, \mathrm{MN}, \mathrm{CB}, \mathrm{LP}$; drafting of the manuscript: $\mathrm{MB}, \mathrm{LP}$; critical revision: $\mathrm{MB}, \mathrm{DM}, \mathrm{MN}, \mathrm{AB}, \mathrm{CB}, \mathrm{LP}$; and supervision: $\mathrm{CB}, \mathrm{LP}$.

Funding This work was supported by grants (to $\mathrm{MB}$ ) from the AOIc2020 (Appel d'Offre Interne COVID-19), from the INSERM (Centre de Recherche UMR 1231), the National Research Agency (ANR) Investissements d'Avenir Grant (ANR-11 LABX-0021-01, Labex Lipstic), the FEDER, the Regional Council of Bourgogne Franche-Comte and by crowdfunding (https://thellie.org/covid-19).

\section{Compliance with ethical standards}

Conflict of interest The authors declare no competing interests. 
Consent to publish All authors consent for publication.

Ethics approval and consent to participate Approval was obtained from the ethics committee (Comité de Protection des Personnes SUD MEDITERRANEE V; 2017-A03404-49). Oral consent was obtained from the patient or their legal representatives.

Publisher's note Springer Nature remains neutral with regard to jurisdictional claims in published maps and institutional affiliations.

\section{References}

1. Popkin BM, Du S, Green WD, Beck MA, Algaith T, Herbst CH, et al. Individuals with obesity and COVID-19: a global perspective on the epidemiology and biological relationships. Obes Rev. 2020;21:e13128.

2. Földi M, Farkas N, Kiss S, Dembrovszky F, Szakács Z, Balaskó $\mathrm{M}$, et al. Visceral adiposity elevates the risk of critical condition in COVID-19: a systematic review and meta-analysis. Obesity (Silver Spring). 2021;29:521-8.

3. Sattar N, McInnes IB, McMurray JJV. Obesity is a risk factor for severe COVID-19 infection: multiple potential mechanisms. Circulation. 2020;142:4-6.

4. Piroth L, Cottenet J, Mariet A-S, Bonniaud P, Blot M, TubertBitter P, et al. Comparison of the characteristics, morbidity, and mortality of COVID-19 and seasonal influenza: a nationwide, population-based retrospective cohort study. Lancet Respir Med. 2021;9:251-9.

5. Rebello CJ, Kirwan JP, Greenway FL. Obesity, the most common comorbidity in SARS-CoV-2: is leptin the link? Int J Obes. 2020;44:1810-7.

6. Weisberg SP, McCann D, Desai M, Rosenbaum M, Leibel RL, Ferrante AW. Obesity is associated with macrophage accumulation in adipose tissue. J Clin Invest. 2003;112:1796-808.
7. Francisco V, Pino J, Campos-Cabaleiro V, Ruiz-Fernández C, Mera A, Gonzalez-Gay MA, et al. Obesity, fat mass and immune system: role for leptin. Front Physiol. 2018;9:640.

8. Blot M, Bour J-B, Quenot JP, Bourredjem A, Nguyen M, Guy $\mathrm{J}$, et al. The dysregulated innate immune response in severe COVID-19 pneumonia that could drive poorer outcome. J Transl Med. 2020;18:457.

9. Huttunen R, Syrjänen J. Obesity and the outcome of infection. Lancet Infect Dis. 2010;10:442-3.

10. Heymsfield SB, Wadden TA. Mechanisms, pathophysiology, and management of obesity. N Engl J Med. 2017;376:1492.

11. van der Voort PHJ, Moser J, Zandstra DF, Muller Kobold AC, Knoester M, Calkhoven CF, et al. Leptin levels in SARS-CoV-2 infection related respiratory failure: a cross-sectional study and a pathophysiological framework on the role of fat tissue. Heliyon. 2020;6:e04696

12. Blot M, Bourredjem A, Binquet C, Piroth L, LYMPHONIE Study Group. Is IL-6 the right target in COVID-19 severe pneumonia? Am J Respir Crit Care Med. 2021;203:139-40.

13. Ubags NDJ, Stapleton RD, Vernooy JHJ, Burg E, Bement J, Hayes CM, et al. Hyperleptinemia is associated with impaired pulmonary host defense. JCI Insight. 2016;1:e82101.

14. Hue S, Beldi-Ferchiou A, Bendib I, Surenaud M, Fourati S, Frapard $\mathrm{T}$, et al. Uncontrolled innate and impaired adaptive immune responses in patients with COVID-19 acute respiratory distress syndrome. Am J Respir Crit Care Med. 2020;202:1509-19.

15. Higham A, Singh D. Increased ACE2 expression in bronchial epithelium of COPD patients who are overweight. Obesity (Silver Spring). 2020;28:1586-9.

16. Honce R, Schultz-Cherry S. Impact of obesity on influenza A virus pathogenesis, immune response, and evolution. Front Immunol. 2019;10:1071.

17. McCartney SA, Kachikis A, Huebner EM, Walker CL, Chandrasekaran S, Adams Waldorf KM. Obesity as a contributor to immunopathology in pregnant and non-pregnant adults with COVID-19. Am J Reprod Immunol. 2020;84:e13320. 